\title{
Significant interlayer coupling in bilayer graphene and double-walled carbon nanotubes: a refinement of obtaining strain in low-dimensional materials
}

\author{
Y. W. Sun,${ }^{1, *}$ D. Gehringer, ${ }^{2}$ D. Holec $,{ }^{2},{ }^{\dagger}$ D. G. Papageorgiou, ${ }^{1}$ O. \\ Fenwick, ${ }^{1}$ S. M. Qureshi, ${ }^{3}$ C. J. Humphreys,${ }^{1}$ and D. J. Dunstan ${ }^{3} \ddagger$ \\ ${ }^{1}$ School of Engineering and Materials Science, Queen Mary University of London, London E1 4NS, United Kingdom \\ ${ }^{2}$ Department of Materials Science, Montanuniversität Leoben, Leoben 8700, Austria \\ ${ }^{3}$ School of Physics and Astronomy, Queen Mary University of London, London E1 4NS, United Kingdom
}

(Dated: January 8, 2022)

\begin{abstract}
This paper solves a longstanding debate: Raman measurements on double-walled carbon nanotubes appear to show that significantly more pressure than expected can be transmitted to the inner tube. We re-interpret those Raman spectra consistently reported in the literature, by assigning the Raman peaks to coupled vibrational modes of both walls, instead of individual contributions from the inner and outer tubes. These coupled vibrational modes are important for the correct interpretation of the Raman shift from strained layered 2D materials (we demonstrate it on bilayer graphene as an example), for researching the mechanical properties, thermal expansion and strain engineering of $2 \mathrm{D}$ materials.
\end{abstract}

\section{INTRODUCTION}

There is a long-standing debate about the response of double-walled carbon nanotubes (DWCNTs) to external pressure.[1] Many papers report shifts in the Raman G-band which are interpreted as the consequence of substantial pressures both on the external tube and on the internal tube.[1-5] However, the individual tube walls are very stiff in-plane (along their axis and around their circumference) because of the strong $s p^{2}$ bonds. [6] Under external pressure, the in-plane strain of an outer tube is small, and so is the reduction of its diameter (i.e. radial strain). Inner and outer tubes interact by weak van der Waals (vdW) forces,[7] and pressure is transmitted to an inner tube via the outer tube. However, due to a small change in distance between the walls (defined by nuclear positions) and a weak inter-wall interaction, the pressure on an inner tube is expected to be close to zero.[8] Taking the Young's modulus of graphene (1 TPa)[6] as the in-plane stiffness of a nanotube, and the out-of-plane stiffness of graphite (40 GPa) $[9]$ as the inter-wall (radial) stiffness in a DWCNT, a crude estimate of the proportion of the external pressure transmitted to an inner tube might be at most about $8 \%$. This is not consistent with the numerous research papers that report two in-plane vibrational modes in the Raman spectra of DWCNTs, the one of lower frequency shifting about two-thirds as fast as the other mode with external pressure. These two modes have been assigned to individual vibrations of the outer and inner tubes, and imply a 60:40 ratio of the pressures supported by the two tubes.[1-5] We solve this puzzle by considering the mechanics of the pressure transmission in more detail, and by re-interpreting the reported Raman spectra as coupled vibrational modes of

\footnotetext{
* yiwei.sun@qmul.ac.uk

$\dagger$ david.holec@unileoben.ac.at

¥d.dunstan@qmul.ac.uk
}

both walls.

This paper is more generally about correctly extracting strain from Raman measurements on layered 2D materials. Research on many fundamental properties of these materials involves application of strain, such as their mechanical properties,[10] thermal expansion, etc.,[11] which can be further tuned by strain for various applications.[12] Also in applications where strain is inevitable, such as a graphene sensor on human skin,[13] an accurate determination of strain is important and, for that, Raman spectroscopy is a powerful and convenient tool, compatible with other simultaneous measurements (e.g. thermal, electrical, mechanical, etc.).[14]

For a layered material having large anisotropy (strong covalent bonds in-plane and weak vdW out-of-plane), one can measure its in-plane phonon frequencies to obtain the in-plane strain (see Eq. 4, which will be introduced later).[14] When the layers are at different strain, the Raman peaks for the in-plane phonons (for example in multilayer graphene, the G-mode at $1580 \mathrm{~cm}^{-1}$ ) split.[15] When bilayer graphene is deposited on a substrate and later strained, the adhesion between the bottom graphene layer and the substrate is usually different from that between the two graphene layers. This results in the strain transferred from the substrate to the bottom layer being different from the strain transferred from the bottom layer to the top layer.[15] The split Raman peaks are commonly assigned to the layers at different strain, respectively,[15] and the coupling between vibrational modes is ignored. This assignment is straightforward and works well when the in-plane vibrational frequencies are significantly different in neighbouring layers (e.g. in heterostructures, isotopes),[16] or the coupling between layers is weak (e.g. with intercalated molecules, functional groups). Here we quantify the impact of the coupling between the vibrational modes of adjacent layers in homostructures to show when it becomes important. 


\section{STRAIN DETERMINATION IN BILAYER GRAPHENE}

We start by relating in-plane phonon frequencies to strain in a simple case, a monolayer graphene. The dynamical equation for the optical in-plane phonon modes is: $[17]$

$$
\sum_{\beta} K_{\alpha \beta} u_{\beta}=\omega^{2} u_{a}
$$

where $\mathbf{u}=\left(u_{1}, u_{2}\right)$ is the displacement of the two carbon atoms ( $\alpha, \beta$ is 1 or 2$)$ in the unit cell, $\omega$ is the phonon frequency, and $K$ is the force constant matrix. Taking a phenomenological approach, we write the force constant matrix as $\left(\begin{array}{cc}\omega_{0}^{2} & 0 \\ 0 & \omega_{0}^{2}\end{array}\right)$, giving one eigenvalue $\omega_{0}^{2}$ for the two degenerate modes of unstrained graphene, along the equivalent in-plane $x$ and $y$-directions, respectively. The zero off-diagonal elements reflect that there is no interaction between in-plane vibrations along orthogonal directions.

In a bilayer graphene, the force constant matrix becomes:[18]

$$
\left(\begin{array}{cccc}
\omega_{0}^{2} & 0 & C & 0 \\
0 & \omega_{0}^{2} & 0 & C \\
C & 0 & \omega_{0}^{2} & 0 \\
0 & C & 0 & \omega_{0}^{2}
\end{array}\right)
$$

where $C$ is for the coupling between vibrations along the same direction in the two layers. For bilayer graphene, or graphite, the in-plane out-of-phase vibration of two layers in a unit cell is a Raman-active $E_{2 g}$ mode and the in-phase is a Raman-inactive $E_{1 u}$ mode.[19] The eigenvalue of the Raman active modes is $\omega_{0}^{2}-C$. This should be diagonalised here, then, the coupling parameter $C$ can be obtained from the difference in frequencies of unstrained monolayer and bilayer graphene (or graphite), ideally measured or calculated in the same experimental or simulation setup.

When there are two layers under different strain, we can introduce the effect of strain by expanding the diagonal elements in Matrix 2 in terms of the corresponding strains.[17] We demonstrate this using an example of an excellent experiment: Wang et al. deposited bilayer graphene on a $\mathrm{SiO}_{2}$ substrate, suspended across a circular hole. The hole was pressurised to put the suspended graphene layers under tension. The top layer was able to slide over the bottom layer, and with sufficient pressure, the bottom layer started to slide over the substrate.[15] Much useful information can be extracted from this experiment. The first step is to relate the recorded Raman frequencies to strain. The shear strength at the graphene- $\mathrm{SiO}_{2}$ interface (1.64 MPa) was reported to be very much larger than between graphene layers $(40 \mathrm{kPa}) .[15]$ Considering the centre of the blister, which is under isotropic biaxial tension, $\varepsilon_{x x}=\varepsilon_{y y}$,
Matrix 3 simplifies to:

$$
\left(\begin{array}{cccc}
\omega_{0}^{2}+A \varepsilon & 0 & C & 0 \\
0 & \omega_{0}^{2}+A \varepsilon & 0 & C \\
C & 0 & \omega_{0}^{2} & 0 \\
0 & C & 0 & \omega_{0}^{2}
\end{array}\right)
$$

where $\varepsilon$ is the biaxial in-plane strain in the bottom layer, and $A$ is the linear coefficient. The strain in graphene is related to its in-plane phonon frequencies by the Grüneisen parameter $\gamma$ and shear deformation potential (SDP):[20]

$$
\frac{\Delta \omega}{\omega_{0}}=\gamma\left(\varepsilon_{x x}+\varepsilon_{y y}\right) \pm \frac{1}{2} S D P\left(\varepsilon_{x x}-\varepsilon_{y y}\right),
$$

from which we obtain $A=4 \gamma \omega_{0}^{2}$. We plot the two distinct eigenvalues of Matrix 3 with isotropic in-plane strain (where the SDP does not need to be considered) in Fig. 1, taking $\omega_{0}=1582 \mathrm{~cm}^{-1}$ for the GM of monolayer graphene and $\sqrt{\omega_{0}^{2}-C}=1578 \mathrm{~cm}^{-1}$ for bilayer graphene in the paper by Wang et al.[15] We also take $\gamma=2$ from Mohiuddin et al.[10] The difference in frequencies at strains less than $0.2 \%$ (requiring a tensile stress of about $2 \mathrm{GPa}$ ) is evident (29\% difference in average slope) between including (black) and ignoring (orange) the coupling. The initial shift rates are -33.0 $\mathrm{cm}^{-1} / \%$ (black) and $-63.5 \mathrm{~cm}^{-1} / \%$ (orange, consistent with that of a monolayer). In other words, if one measures Raman frequencies of such a bilayer, choses a linear shift rate with biaxial strain from a previous experiment on monolayer graphene (e.g. $-63.5 \mathrm{~cm}^{-1} / \%$ from Ref. 10 ) and does not consider the coupling, an error up to $29 \%$ in the obtained strain (for less than $0.2 \%$ strain) is then expected. Beyond a strain $\sim 0.3 \%$ of one layer, the in-plane vibrational frequencies in the two layers become increasingly different, and therefore the impact of the coupling becomes insignificant. The originally Raman-inactive inphase vibration becomes Raman-active because of the break of the symmetry, and its frequency approaches the GM of the top layer at zero strain alone, whereas the frequency of the out-of-phase vibration approaches that of the strained layer.

\section{STRAIN DETERMINATION IN DWCNTS}

There are additional complexities in DWCNTs, from the tube wall curvature. The pressure transmitted to an inner tube from an external compression should be small, because of the large in-plane stiffness of the outer tube and the weak inter-tube interaction. In this section, we first quantify the small amount of pressure transmitted to the inner tube from the mechanical elastic behaviour, and estimate the contribution from the squeezing of $\pi$ orbitals, using density functional theory (DFT). We then provide a corrected assignment of the measured Raman peaks, following the approach introduced above, that accounts for the coupling of the vibration between tube walls, to solve the long-standing debate. 


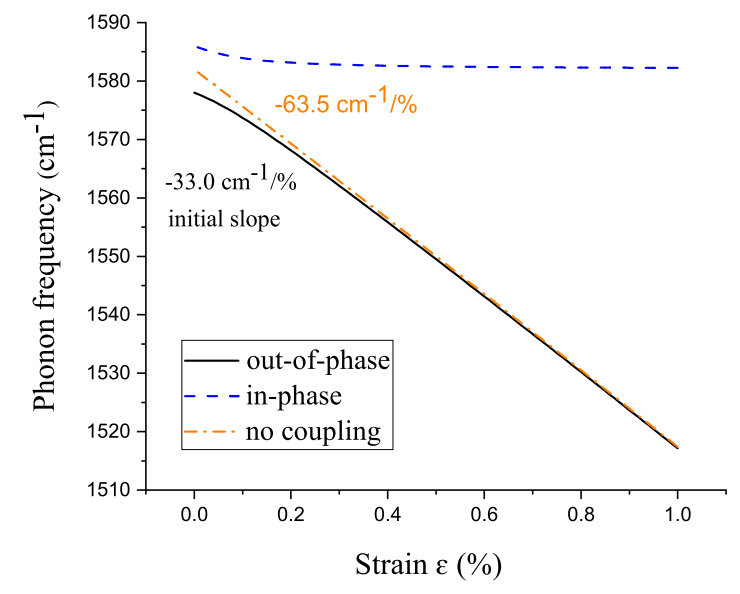

FIG. 1. Calculated phonon frequencies of the coupled in-plane vibrations in bilayer graphene are plotted as a function of tensile isotropic strain in one layer whereas there is no strain in the other. The frequencies of the two-plane out-of-phase vibration (Raman-active, black solid) and two-plane in-phase vibration (Raman-inactive at zero strain, blue dashed) are the square root of the eigenvalues of Matrix 3. The orange dash-dotted line is obtained by ignoring the unstrained layer (equivalently a strained monolayer). The vibrations in the two layers decouple when the black and orange lines overlap at strains more than $0.5 \%$. The initial shift rates of the phonon frequencies of the Raman-active modes with isotropic strain are presented.

\section{A. Anisotropic and Inhomogeneous Thick-Walled Cylinders}

Two models of DWCNTs under pressure are presented. In the first model, graphene is considered as a highly anisotropic continuum material, very stiff in plane and very soft in the third direction. Rolled up as a nanotube, it is soft in the radial direction. In the second model, graphene is considered as a very stiff $2 \mathrm{D}$ sheet, with soft isotropic material on both sides (corresponding to the $\pi$ orbitals). For both models, we require the classic Lamé equations for thick-walled tubes under pressure.[21] We begin with the derivation of these equations, modified for an anisotropic material, as this does not appear to have been given previously.

\section{Lamé equations for anisotropic material}

Consider a thick-walled tube under pressure (external or internal or both); by symmetry the material of the tube is displaced radially by an amount $u(r)$. There is no tangential displacement. The axial stress will be considered later, but generally it is half the hoop stress. Then we have hoop, radial and axial strains and stresses, defined and related by,

$$
\begin{array}{r}
\varepsilon_{H}(r)=\frac{u(r)}{r}=\frac{\sigma_{H}(r)}{Y}-\frac{\nu \sigma_{A}(r)}{Y}-\frac{\nu \sigma_{R}(r)}{Y_{\perp}} \\
\varepsilon_{R}(r)=\frac{d u(r)}{d r}=\frac{\sigma_{R}(r)}{Y_{\perp}}-\frac{\nu_{\perp} \sigma_{H}(r)}{Y}-\frac{\nu_{\perp} \sigma_{A}(r)}{Y} \\
\varepsilon_{A}(r)=\frac{\sigma_{A}(r)}{Y}-\frac{\nu \sigma_{H}(r)}{Y}-\frac{\nu_{\perp} \sigma_{R}(r)}{Y_{\perp}}
\end{array}
$$

We simplify immediately by assuming the elastic constant $\nu_{\perp}=0$, as for graphite. Then from $\varepsilon_{R}$ and from $\varepsilon_{H}^{\prime}$ we have two expressions for $u^{\prime}$ which we can equate (and multiply by $Y$ ), so that,

$$
\frac{Y \sigma_{R}}{Y_{\perp}}-\sigma_{H}-r \sigma_{H}^{\prime}+\nu \sigma_{A}+r \sigma_{R}^{\prime}=0
$$

The axial strain is everywhere the same, as it expresses the change in length of the tubes under pressure, so

$$
Y \varepsilon^{\prime(r)}=\sigma_{A}^{\prime}-\nu \sigma_{H}^{\prime}=0
$$

Using this to eliminate $\sigma_{A}^{\prime}$, together with the equation of equilibrium (force balance) in polar co-ordinates, $\sigma_{H}=\sigma_{R}+\sigma_{R}^{\prime}$, to eliminate $\sigma_{H}$, Eq. 6 becomes a lengthy and intractable expression including terms in $\sigma_{A}$ and the parameters describing the anisotropy. At this point, if the material is isotropic (i.e. $Y_{\perp}=Y$ and $\nu_{\perp}=\nu$ ) a very simple expression is obtained. If, on the other hand, we retain $Y_{\perp}$ and put $\nu_{\perp}=\nu=0$, we obtain

$$
\left(\frac{Y \sigma_{R}}{Y_{\perp}}-1\right) \sigma_{R}-r \sigma_{R}^{\prime}+r \sigma_{H}^{\prime}=0
$$

For $Y_{\perp}=Y$ this simplifies to the simple isotropic equation which may be integrated, and the Lamé equations obtained directly. Here, we can substitute for $\sigma_{H}^{\prime}$ from the differential of the equilibrium equation, to obtain the differential equation in $\sigma_{R}$,

$$
\left(\frac{Y \sigma_{R}}{Y_{\perp}}-1\right) \sigma_{R}-r \sigma_{R}^{\prime}-2 r \sigma_{R}^{\prime}+r \sigma_{R}^{\prime \prime}
$$

This has the solution,

$$
\sigma_{R}(r)=C_{1} r^{-1-\sqrt{Y / Y_{\perp}}}+C_{2} r^{-1+\sqrt{Y / Y_{\perp}}}
$$

Putting in the boundary conditions for a tube with an internal radius $a$ and pressure $P_{a}$ and external radius $b$ and pressure $P_{b}$, solving Eq. 9 for $C_{1}$ and $C_{2}$ with $\sigma_{R}(a)=-P_{a}$ and $\sigma_{R}(b)=-P_{b}$ gives

$$
\begin{array}{r}
C_{1}=(a b)^{\sqrt{Y / Y_{\perp}}}\left(\frac{a b^{\sqrt{Y / Y_{\perp}}} P_{a}-b a \sqrt{Y / Y_{\perp}} P_{b}}{b^{2 \sqrt{Y / Y_{\perp}}}-a^{2 \sqrt{Y / Y_{\perp}}}}\right) \\
C_{2}=\frac{a^{1+\sqrt{Y / Y_{\perp}}} P_{a}-b^{1+\sqrt{Y / Y_{\perp}}} P_{b}}{b^{2 \sqrt{Y / Y_{\perp}}}-a^{2 \sqrt{Y / Y_{\perp}}}}
\end{array}
$$

Then the equilibrium equation gives

$$
\sigma_{H}(r)=\sqrt{Y / Y_{\perp}} r^{-1-\sqrt{Y / Y_{\perp}}}\left(C_{1}+C_{2} r^{2 \sqrt{Y / Y_{\perp}}}\right)
$$




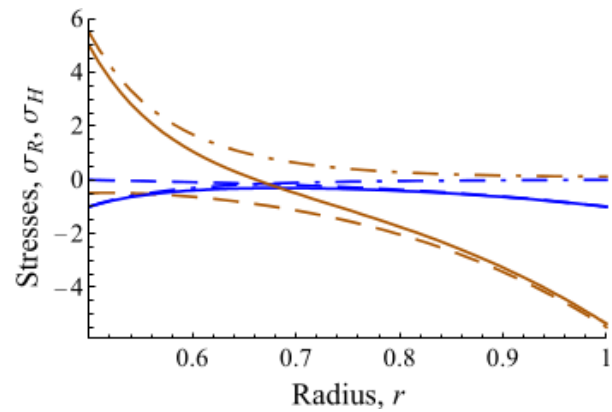

FIG. 2. The radial (blue curves $0<y<1$ ) and hoop stresses (brown curves to large positive and negative values) are plotted against radius for a material as anisotropic as graphene, for internal pressure $P_{a}=0$ external pressure $P_{b}=1$ (dashed curves), for $P_{a}=1, P_{b}=0$ (chain-dotted curves) and for hydrostatic pressure $P_{a}=1, P_{b}=1$ (solid curves). The outer diameter is unity, and the inner diameter is $1 / 2$.

Thick-Wall Model: These solutions give stresses very different from isotropic material. In Fig. 2 they are plotted for an internal radius of $a=1 / 2$, external radius $\mathrm{b}=$ 1 , for $Y / Y_{\perp}=30$ (as for graphene) and for three cases of internal and external pressures of 0 and 1, 1 and 0 , and 1 and 1 . The hoop stresses near the inner and outer surfaces are much larger than in the isotropic case.

The anisotropy has resulted in a separation of the responses of the material. The material near the inner bore responds to the inner pressure, and the material near the outer surface responds to the external pressure, each nearly independently of the other. This is most dramatically seen with equal inner and outer pressures (Fig. 2). In this case, an isotropic material would have simply hydrostatic pressure throughout, with $\sigma_{R}=\sigma_{H}=-1 \mathrm{ev}-$ erywhere. The anisotropic material has a large tensile $\sigma_{H}$ near the inner bore, large compressive $\sigma_{H}$ near the outer surface, and both radial and compressive stresses dropping to low values near zero in between, in material that is shielded from both internal and external pressures by the large hoop stiffness that carry most of the load near the inner and outer surfaces.

The reverse anisotropy is less dramatic. With a tangential stiffness 30 times less than the radial stiffness, all stresses are much closer to the isotropic case, as shown in Fig. 3. Perhaps the only observation of interest is that in the hydrostatic case the radial stress rises a little above the applied hydrostatic pressure.

Approximations: The effects of the approximations we made to get these solutions should be considered. It is rather remarkable that the Poisson's ratio of an isotropic material does not enter into Lamé's equation for the stresses, but we may use that as a justification for supposing that the Poisson's ratios for the anisotropic material are similarly unimportant. Certainly, as far as the gross features of Fig. 2 and Fig. 3 are concerned, there is no reason to believe that Poisson's ratios would affect the stresses. Of course, in calculating the strains, the Poisson's ratios will have some effect.

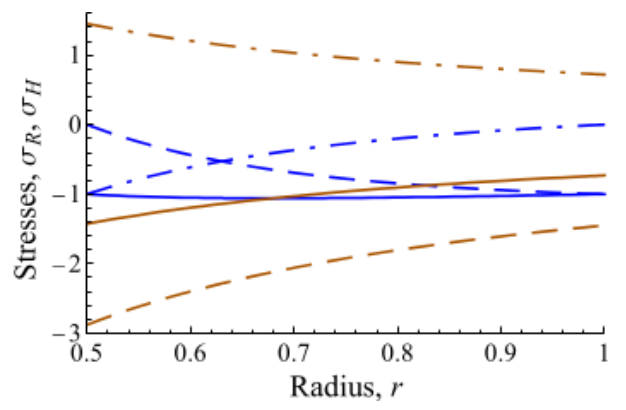

FIG. 3. As Fig. 2, but for a material with radial stiffness 30 times the tangential stiffness.

Multiwalled tubes: In this model, the stresses are unaffected by the tube being single walled or multiwalled (unless there are interface gaps or interference fits (pressures) at ambient pressure, but this not considered here). The question, how much of an external pressure is supported by the outer tube and how much by the inner tube - and what would be the effects on the Raman spectra of inner and outer tubes - can be addressed by calculating the hoop strain $\varepsilon_{H}(r)$ and averaging it over the inner tube, and over the outer tube. A good approximation may be found by averaging the hoop stress instead. However, most directly, the radial stress at the interface should simply be the pressure exerted by the outer tube on the inner. For a DWCNT under external pressure $P_{b}=1, P_{a}=0$, with the outer tube $s p^{2}$ diameter $1.65 \mathrm{~nm}$, inner tube diameter $0.95 \mathrm{~nm}$, and taking the graphene thickness as $0.35 \mathrm{~nm}$, the hoop stress averages for the outer tube and inner tube are respectively -2.58 and -0.273 (from Eq. 11 and the boundary condition), so that 0.095 of the pressure is supported by the inner tube. The radial stress at the interface is -0.147 . We consider that the latter is the correct value, as the two tubes have different ratios of wall thickness to diameter, and this should affect the relationship between pressure and hoop stress (less stress for the same pressure when the tube diameter is smaller relative to the wall thickness).

\section{Isotropic 3D $\pi$-material and $2 D$ sp2 material}

Thin-Wall Model: Here we use the Lamé equations for the same DWCNT modelled as three soft isotropic thick-walled tubes, separated by two stiff $2 \mathrm{D}$ tubes. In particular, we use the solutions, Eq. 9, 10 and 11 with $Y / Y_{\perp}=1$. We calculate the hoop strain $\varepsilon_{H}(r)$ of the outer $\pi$-orbital tube with $P_{b}=P_{1}=1 \mathrm{GPa}$ and $P_{a}=P_{2}$ kept as a variable. Then we calculate $\varepsilon_{H}$ of the outer tube with its internal pressure $P_{3}$ as a variable. Equating these two hoop strains at $r=0.65 \mathrm{~nm}$ lets us eliminate $P_{2}$, expressing it as a function of $P_{1}$ and $P_{3}$. Continuing across in this way we eliminate the pressures $P_{3}, P_{4}$ and $P_{5}$ until we reach the inner bore of the inner tube, with the variable pressure $P_{6}$. Equating this to zero solves for 

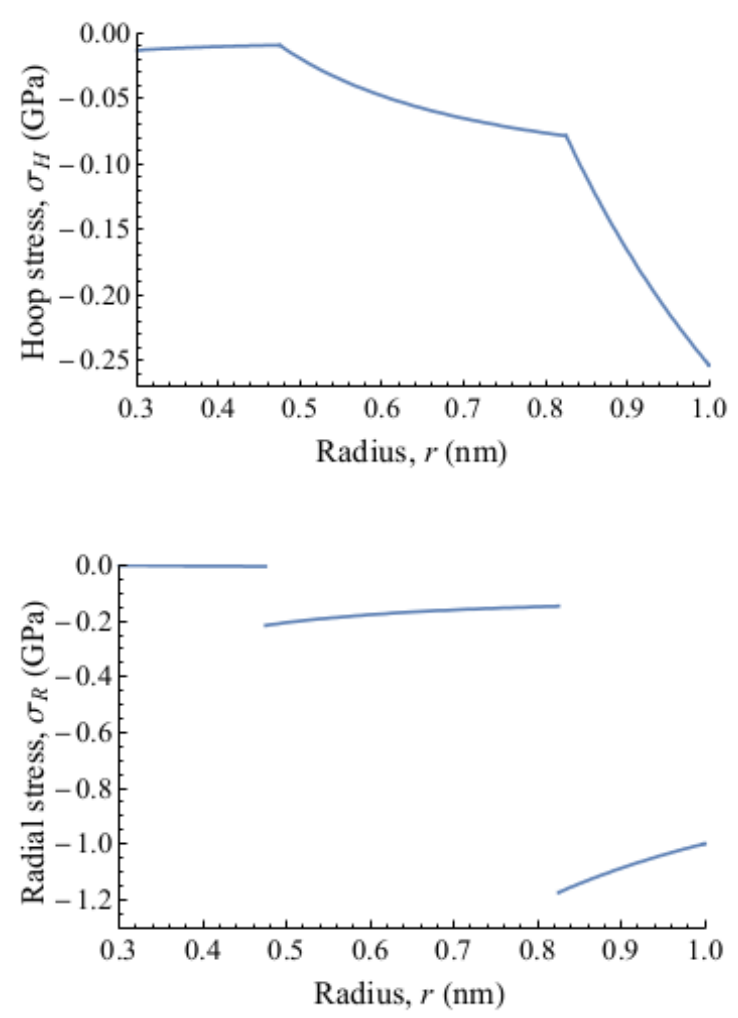

FIG. 4. The hoop (top graph) and the radial (bottom graph) stresses in the 3D material are plotted against radius, from outer surface of the outer tube to inner bore of the inner tube.

all the pressures. They are, $P_{6}=0, P_{5}=4.01 \mathrm{MPa}$, $P_{4}=216 \mathrm{MPa}, P_{3}=147 \mathrm{MPa}, P_{2}=1.18 \mathrm{GPa}$ and $P_{1}=1 \mathrm{GPa}$. The pressure on the inner tube is the radial stress at the interface at $r=0.65 \mathrm{~nm}$, which is $168 \mathrm{MPa}$ $\left(P_{3^{1 / 2}}\right)$. The hoop and radial stresses across the radius of a DWCNT are plotted in Fig. 4.

Note that the 2D tubes are under substantially higher pressures than the pressures applied to the outside of their $\pi$-orbitals. A physical interpretation of this effect is that under hydrostatic pressure the soft material contracts much more that the stiff $2 \mathrm{D}$ tubes. Consequently, to keep its inner radius the same as the radius of the $2 \mathrm{D}$ tubes, it has to be stretched - and the softer it is, the more it has to be stretched, so the force is largely independent of its elastic modulus - and this is sufficient to return the hoop strain much closer to zero. This is a combination of hydrostatic pressure plus hoop tension. The hoop tension then applies extra pressure above the hydrostatic pressure to the $2 \mathrm{D}$ tube inside it.

\section{B. DFT estimate of pressure transmission in DWCNTs}

We employed DFT[22] to examine how much pressure could be transmitted to the inner tube via the squeez- ing of $\pi$-orbitals under external compression, in addition to the above classical mechanics analysis. Under external pressure, the $\pi$-orbitals of an outer tube are expected to be squeezed inwards through the tube wall, which increases the overlap of $\pi$-electronic orbitals between inner and outer tubes, which results a pressure being transmitted to the inner tubes. The calculation setup was the same as in our previous work on graphite and bilayer graphene.[23] We modelled three layers of Bernal stacked graphene. We fixed the position of the bottom two layers and displaced the top layer towards the bottom two. The bottom two layers simulated a DWCNT with interlayer distance fixed at $3.4 \AA$. External pressure to the bilayer graphene at the bottom was applied by the approaching top layer.

We calculated the stress on the bottom layer at each position of the top layer (defined by its distance to the fixed middle layer), as shown in Fig. 5 (a). $3.4 \AA$ is slightly smaller than the calculated equilibrium interlayer distance and therefore the bottom layer is under a small repulsive stress. Our interest is the change of it with the displacement of the top layer. When the top layer was displaced from 3.4 to $2.5 \AA$ close to the middle layer, this results in a pressure applied to the middle layer of about $50 \mathrm{GPa}$, whereas the repulsive stress on the bottom layer was reduced by only $0.1 \mathrm{GPa}$. This indicates that the stress transmitted through the middle layer (an outer tube) to the bottom layer (an inner tube) by the squeeze of $\pi$-electrons is negligible. Furthermore, it was not repulsion, but a small attraction that was applied to the bottom layer. This can be explained by the change in valence charge at each interlayer, as shown in Fig. 5 (b). The valence charge between the top and middle layer was reduced as expected, when the top layer approached the middle. These charges were evenly squeezed to the bottom-middle interlayer region, and outside the top layer. Consequentially, the bottom layer had an overall negative charge, and was therefore attracted by the middle layer of positive charge, as it loses more charges on one side (above) than gaining on the other (below). So it seems that the only mechanism by which pressure is transmitted to the inner tube is through the mechanical elastic behaviour of Sec. III A.

\section{Inter-wall vibrational coupling in DWCNTs}

Placing the model of Sec. III A 2 under hydrostatic pressure, we obtained the radial and hoop stress $\sigma_{R}(r)$ and $\sigma_{H}(r)$. This model provides refined pressure transmission to the inner tube and much clearer underlying physics, compared to the existing understanding. [1, 24]

The axial stress is fully supported by the two stiff tubes. Using the three stress components, and the compliance constants $s_{11}$ and $s_{12}$ of graphene, the hoop and axial strains are then obtained for the stiff tubes.[24] The hoop strain of the inner tube is typically 0.1 to 0.2 of the strain of the outer tube. This cannot explain the 
(a)

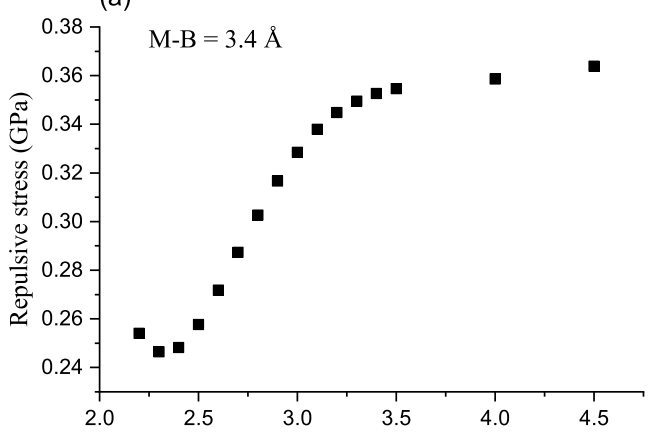

(b)

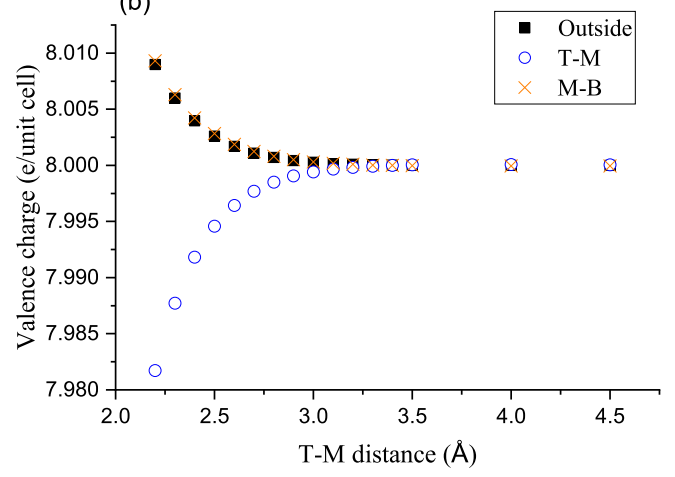

FIG. 5. In a trilayer graphene, the top layer is displaced towards the fixed bottom two layers with an interlayer distance of $3.4 \AA$. (a) The repulsive stress on the bottom layer is plotted with the distance between the top and middle layer. (b) The valence charge between the top and middle layer (blue open circles), between the middle and bottom layer (orange crosses) and outside the top layer (black solid squares) in a unit cell is plotted with the distance between the top and middle layer.

$$
\left(\begin{array}{cc}
\omega_{A}^{2}+A \varepsilon_{A o}+B \varepsilon_{T o} & 0 \\
0 & \omega_{o}^{2}+B \varepsilon_{A}+A \varepsilon_{T o} \\
C & 0 \\
0 & C
\end{array}\right.
$$

The subscript $A$ is along the tube axis, $T$ is around the circumference, $o$ is for an outer tube and $i$ is for an inner tube. $A$ and $B$ can be obtained similarly as in a bilayer graphene with the same $\gamma=2$ and $S D P=1$. $\omega_{o}$ and $\omega_{i}$ depend on the diameter of outer and inner tubes, respectively. The axial strains of the inner and outer tubes are the same, because the ends remain together.

We note that the in-plane elastic stiffness of a nanotube surface is nearly isotropic (i.e. $s_{11}=s_{22}, s_{12}=s_{21}$ ) despite a curved tube wall, as the difference of a few tens of $\mathrm{cm}^{-1}$ between the $G^{+}$(along tube axis) and $G^{-}$ (along tube circumference) vibrational frequencies puts an upper limit of only a few percent on any difference in the stiffness along the two in-plane directions. [25] With $-s_{12} / s_{11}=0.13$ and $s_{11}+s_{12}=1 / 1250 \mathrm{GPa}^{-1},[24]$ we reported shift rates of the GMs with pressure in DWCNTs, if the two modes were assigned to inner and outer tubes correspondingly. Below we propose and explain an interpretation that accommodates the small pressure transmission to the inner tube, for the large amounts of consistent published data on DWCNTs.

For a DWCNT, Matrix 3 becomes,

$$
\left.\begin{array}{cc}
C & 0 \\
0 & C \\
\omega_{A}^{2}+A \varepsilon_{A}+B \varepsilon_{T i} & 0 \\
0 & \omega_{i}^{2}+B \varepsilon_{A i}+A \varepsilon_{T i}
\end{array}\right)
$$

plot the frequencies (square root of the four eigenvalues of Matrix 12) as a function of external pressure in Fig. 6, taking $\omega_{a x}=1591 \mathrm{~cm}^{-1}, \omega_{\text {out }}=1574 \mathrm{~cm}^{-1}$, and $\omega_{i n}=1543 \mathrm{~cm}^{-1}$ for a DWCNT with an outer diameter of $1 \mathrm{~nm}$, the same as in the above stress analysis, using the established relationship between tube diameters and $\omega_{T \cdot} \cdot[25]$

The key information we extract from Fig. 6 is as follows. The in-phase vibrational mode of the two walls along the axial direction is Raman-inactive at zero pressure. But with less coupling between the tube walls under increasing pressure, the Raman intensity of this mode increases and its frequency approaches the $\mathrm{G}^{+}$of the outer tube. These two frequencies are the same when the inplane vibrations in the outer and inner tubes decouple at 


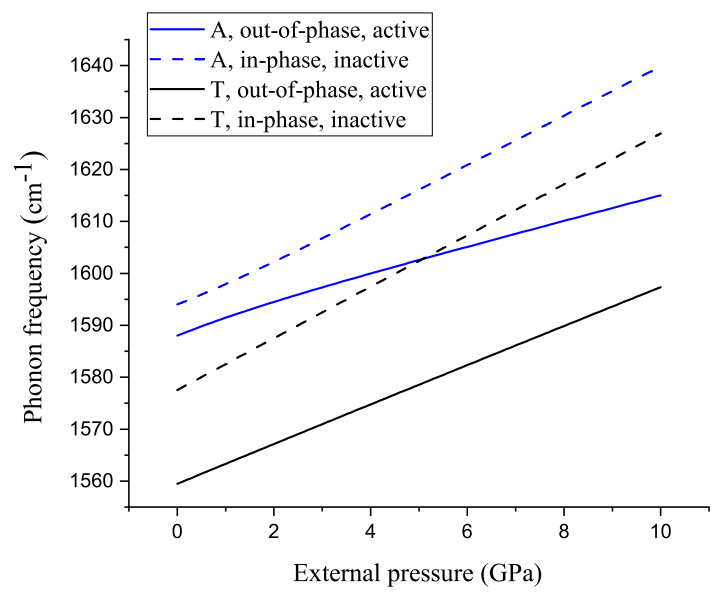

FIG. 6. Phonon frequencies of the coupled in-plane vibrations in a DWCNT (outer radius $1 \mathrm{~nm}$ of the outmost soft tube as an example) are plotted as a function of external hydrostatic pressure. The frequencies are square root of the four eigenvalues of Matrix 12, the corresponding eigenmodes to which are coupled vibrations of the outer and inner tubes. Whether the coupled vibration is axial or tangential, and in-phase or out-of-phase, are labeled. The dashed lines are for Ramaninactive modes when the inner and outer tubes are coupled. The solid lines are for Raman-active modes.

roughly over $5 \mathrm{GPa}$ (see Fig. 1 for the decoupling condition). The out-of-phase vibrational mode of the two walls along the axial direction is a Raman-active mode, and its frequency approaches the $\mathrm{G}^{+}$of the inner tube when the vibration of the two walls decouples. Along the tangential direction, because of the very different wall curvature between inner and outer tubes, the vibration is already decoupled at zero pressure and therefore the frequencies of the out-of-phase and in-phase mode are very close to the $\mathrm{G}^{-}$of the outer and inner tubes, respectively. In an experimental Raman spectrum, at low pressure there should be a main peak of the axial outof-phase mode at higher frequency, and weak tangential peak $(\mathrm{s})$ at lower frequency depending on the quality of the spectrum; at high pressure (e.g. above $5 \mathrm{GPa}$ ), there should be a broad profile consisting of three peaks in a relatively small frequency range (axial in-phase, tangential in-phase, and axial out-of-phase modes) at higher fre- quency, and a separate single peak of the tangential outof-phase mode at lower frequency. These are consistent with the commonly observed spectra in the literature.[15] As seen from Fig. 6, the shift rates with pressure of the vibrations along the axial direction are 4.4 and $2.9 \mathrm{~cm}^{-1} \mathrm{GPa}^{-1}$, for in- and out-of-phase modes, respectively. The shift rates for the tangential modes are 5.0 and $3.81 \mathrm{~cm}^{-1} \mathrm{GPa}^{-1}$, respectively. The reported large shift of the lower-frequency Raman in-plane mode of a DWCNT with pressure is due to the mis-assignment of peaks, and therefore no longer suggests a puzzling large strain of the inner tube. Hence the long-standing debate on this issue has been solved.

\section{CONCLUSION}

In conclusion, we demonstrate the significant impact of interlayer coupling between vibrational modes on obtaining the strain of layered 2D materials from Raman measurements. In a representative example, an error of about $30 \%$ (for up to $0.2 \%$ isotropic strain) is expected in the strain determination in bilayer graphene, if this coupling is ignored. We also solve a long-standing debate concerning Raman measurements under high pressure on DWCNTs, by interpreting the Raman spectra in terms of the coupled vibrations of both walls along the tube axis and circumference, rather than to the separate vibrations of the inner and outer tubes. The widely-reported large shifts of both in-plane Raman peaks in DWCNTs with external pressure, no longer contradicts the reasonable expectation that the strain of the inner tube should be considerably smaller than that of the outer tube. Finally, we quantify the amount of the pressure that can be transmitted to the inner tube by squeezing the electronic orbitals and find it to be negligible.

\section{ACKNOWLEDGMENTS}

DGP acknowledges the support from 'Graphene Core 3' GA: 881603 which is implemented under the EUHorizon 2020 Research \& Innovation Actions (RIA) and is financially supported by EC-financed parts of the Graphene Flagship.
[1] P. Puech, H. Hubel, D. J. Dunstan, R. R. Bacsa, C. Laurent, and W. S. Bacsa, Discontinuous tangential stress in double wall carbon nanotubes, Phys. Rev. Lett. 93, 095506 (2004).

[2] A. L. Aguiar, E. B. Barros, R. B. Capaz, A. G. Souza Filho, P. T. C. Freire, J. M. Filho, D. Machon, C. Caillier, Y. A. Kim, H. Muramatsu, M. Endo, and A. San-Miguel, Pressure-induced collapse in doublewalled carbon nanotubes: Chemical and mechanical screening effects, J. Phys. Chem. C 115, 5378 (2011).

[3] J. González, C. Power, E. Belandria, J. Jorge, F. Gonzalez-Jimenez, M. Millot, S. Nanot, J. M. Broto, and E. Flahaut, Pressure dependence of raman modes in double wall carbon nanotubes filled with $\alpha$-fe, High Pressure Research 28, 577 (2008).

[4] P. Puech, A. Ghandour, A. Sapelkin, C. Tinguely, E. Flahaut, D. J. Dunstan, and W. Bacsa, Raman g band in double-wall carbon nanotubes combining $\mathrm{p}$ doping and 
high pressure, Phys. Rev. B 78, 045413 (2008).

[5] J. Arvanitidis, D. Christofilos, K. Papagelis, K. S. Andrikopoulos, T. Takenobu, Y. Iwasa, H. Kataura, S. Ves, and G. A. Kourouklis, Pressure screening in the interior of primary shells in double-wall carbon nanotubes, Phys. Rev. B 71, 125404 (2005).

[6] C. Lee, X. Wei, J. W. Kysar, and J. Hone, Measurement of the elastic properties and intrinsic strength of monolayer graphene, Science 321, 385 (2008).

[7] T. C. Hirschmann, P. T. Araujo, H. Muramatsu, J. F. Rodriguez-Nieva, M. Seifert, K. Nielsch, Y. A. Kim, and M. S. Dresselhaus, Role of intertube interactions in double- and triple-walled carbon nanotubes, ACS Nano 8, 1330 (2014).

[8] Y. Sun and D. J. Dunstan, Carbon nanotubes under pressure, in Structure and Multiscale Mechanics of Carbon Nanomaterials, CISM International Centre for Mechanical Sciences, edited by O. Paris (Springer, Vienna, 2016) pp. 99-134.

[9] M. Hanfland, H. Beister, and K. Syassen, Graphite under pressure: Equation of state and first-order raman modes, Phys. Rev. B 39, 12598 (1989).

[10] T. M. G. Mohiuddin, A. Lombardo, R. R. Nair, A. Bonetti, G. Savini, R. Jalil, N. Bonini, D. M. Basko, C. Galiotis, N. Marzari, K. S. Novoselov, A. K. Geim, and A. C. Ferrari, Uniaxial strain in graphene by raman spectroscopy: G peak splitting, gruneisen parameters, and sample orientation, Phys. Rev. B 79, 205433 (2009).

[11] D. Yoon, Y.-W. Son, and H. Cheong, Negative thermal expansion coefficient of graphene measured by raman spectroscopy, Nano Lett. 11, 3227 (2011).

[12] C. Si, Z. Sun, and F. Liu, Strain engineering of graphene: a review, Nanoscale 8, 3207 (2016).

[13] P. Miao, J. Wang, C. Zhang, M. Sun, S. Cheng, and H. Liu, Graphene nanostructure-based tactile sensors for electronic skin applications, Nano-Micro Lett. 11, 71 (2019).

[14] J.-B. Wu, M.-L. Lin, X. Cong, H.-N. Liu, and P.-H. Tan, Raman spectroscopy of graphene-based materials and its applications in related devices, Chem. Soc. Rev. 47, 1822 (2018).
[15] G. Wang, Z. Dai, Y. Wang, P. Tan, L. Liu, Z. Xu, Y. Wei, R. Huang, and Z. Zhang, Measuring interlayer shear stress in bilayer graphene, Phys. Rev. Lett. 119, 036101 (2017).

[16] A. Forestier, F. Balima, C. Bousige, G. d. S. Pinheiro, R. Fulcrand, M. Kalbáč, D. Machon, and A. San-Miguel, Strain and piezo-doping mismatch between graphene layers, J. Phys. Chem. C 124, 11193 (2020).

[17] M. Huang, H. Yan, C. Chen, D. Song, T. F. Heinz, and J. Hone, Phonon softening and crystallographic orientation of strained graphene studied by raman spectroscopy, PNAS 106, 7304 (2009).

[18] Y. W. Sun, D. Holec, and D. J. Dunstan, Graphite under uniaxial compression along the c axis: A parameter to relate out-of-plane strain to in-plane phonon frequency, Phys. Rev. B 92, 094108 (2015).

[19] S. Reich and C. Thomsen, Raman spectroscopy of graphite, Philosophical Transactions of the Royal Society of London. Series A: Mathematical, Physical and Engineering Sciences 362, 2271 (2004).

[20] C. Thomsen, S. Reich, and P. Ordejón, Ab initio determination of the phonon deformation potentials of graphene, Phys. Rev. B 65, 073403 (2002).

[21] G. Lame, Sur les surfaces isothermes dans les corps homogènes en équilibre de température, Journal de mathématiques pures et appliquées 2, 147 (1837).

[22] G. Kresse and J. Furthmüller, Efficient iterative schemes for ab initio total-energy calculations using a plane-wave basis set, Phys. Rev. B 54, 11169 (1996).

[23] Y. W. Sun, D. Holec, D. Gehringer, O. Fenwick, D. J. Dunstan, and C. J. Humphreys, Unexpected softness of bilayer graphene and softening of a-a stacked graphene layers, Phys. Rev. B 101, 125421 (2020).

[24] A. J. Ghandour, I. F. Crowe, J. E. Proctor, Y. W. Sun, M. P. Halsall, I. Hernandez, A. Sapelkin, and D. J. Dunstan, Pressure coefficients of raman modes of carbon nanotubes resolved by chirality: Environmental effect on graphene sheet, Phys. Rev. B 87, 085416 (2013).

[25] A. Jorio, A. G. Souza Filho, G. Dresselhaus, M. S. Dresselhaus, A. K. Swan, M. S. Ünlü, B. B. Goldberg, M. A. Pimenta, J. H. Hafner, C. M. Lieber, and R. Saito, Gband resonant raman study of 62 isolated single-wall carbon nanotubes, Phys. Rev. B 65, 155412 (2002). 